\title{
Adverse Effect of Prolonged Formalin Fixation on DNA Histograms in Paraffin-Embedded Tissue
}

\author{
Jiro Fujimoto, Nobutaka Ichikawa, Masaharu Takeuchi, Hidenao Yamamoto, \\ Takahiro Ueki, Tadamichi Hirano and Eizo Okamoto
}

First Department of Surgery, Hyogo College of Medicine, Nishinomiya, 663-8501

Received for publication August 7, 1996 and in revised form April 30, 1998

In a retrospective analysis of 174 formalin fixed, paraffin-embedded, hepatocellular carcinoma tissue samples, the correlation between the coefficient of variation (CV) as determined by flow cytometry and the duration of formalin fixation (DFF) was investigated. In addition a potential adverse effect of prolonged formalin fixation on $\mathrm{CV}$ and fluorescence intensity in paraffin-embedded liver tissue was also investigated prospectively in samples from four patients in which the DFF varied from three days to four weeks. In the retrospective study, there was a significant linear correlation between the CV and DFF ( $p<0.001)$, with the $C V$ increasing as the DFF became prolonged. The propor- tion of samples in which the CV was less than 10.0 was significantly greater $(p<0.01)$ in samples in which the DFF was less than two weeks. In the prospective study, a decrease in fluorescence intensity was observed as the DFF increased. Following two weeks of formalin fixation the fluorescence intensity decreased to $38.1 \%$ of the intensity observed in fresh samples. The mean $\mathrm{CV}$ was greater than $\mathbf{1 0 . 0}$ in the samples in which the DFF exceeded two weeks. It is concluded that prolonged formalin fixation has an adverse effect on CV. The DFF for flow cytometric analysis of paraffin-embedded tissue should be no greater than two weeks.

Key words: DNA-ploidy, Paraffin-embedded tissue, Formalin

\section{Introduction}

Numerous studies have correlated DNA ploidy with prognosis in cancer patients $[3,5,8,9,13,15]$. Hedley et al. [7] developed a technique which permitted the retrospective flow cytometric analysis of nuclei released from paraffin-embedded tissue. Since this initial report clinical application of this procedure has steadily increased [6]. A disadvantage, however, of using paraffin-embedded tissue in flow cytometric analysis is that a wider CV may result in an inability to detect a small near-diploid aneuploid populations in some samples. In a previous report we experienced this technical difficulty in $22 \%$ of hepatocellular carcinoma (HCC) tissue samples [5]. Previous studies have also concluded that the relative fluorescence intensity decreases after formalin fixation [2, 12]. In this report we determined the $C V$ and fluorescence intensity as a function of the DFF in paraffin-embedded tissue. We asked whether the $\mathrm{CV}$ increased after formalin

Correspondence to: Dr. Jiro Fujimoto, First Department of Surgery, Hyogo College of Medicine, 1-1 Mukogawacho, Nishinomiya, Hyogo 663-8501, Japan. fixation, and, if so, what is the maximum length of fixation time permissible for accurate flow cytometric DNA analysis?

\section{Materials and Methods}

\section{Retrospective study}

DNA ploidy analysis was performed using flow cytometry on specimens from 174 patients who underwent hepatic resection for $\mathrm{HCC}$ during the period from January 1981 to December 1991. The mean age of the patients was 56 years (range 22-75), 89\% of whom were male. Representative samples of the tumor were fixed in $10 \%$ neutral buffered formalin, embedded in paraffin, sectioned, and stained with hematoxylin and eosin. The original histologic preparations from the tumor were reviewed by senior pathologists and the diagnosis of $\mathrm{HCC}$ was confirmed. Flow cytometric DNA analysis was performed on nuclei isolated from paraffin sections by the method of Hedley et al [7]. Isolated nuclei were stained with propidium iodide by the method of Vindelov et al [16]. The samples were analyzed with a FACScan analyzer (Becton and Dickinson, Sunnyvale, CA) equipped with a 
$15 \mathrm{~mW}$ argon ion laser operating at $488 \mathrm{~nm}$. For each sample, 20,000 nuclei were analyzed. Data were analyzed using the Cell fit program (Becton and Dickinson, Sunnyvale, CA).

Duration of formalin fixation (DFF) was reviewed for each sample by pathologic reports which included the date of operation and the date when paraffin-embedded sections were made. The coefficient of variation was calculated for all GOG1 peaks. Samples in which the CV was more than 20.0 and, in which the hitogram revealed a diffuse scattering in all channels, were classified as uninterpretable. A potential correlation between the $\mathrm{CV}$ and the DFF was therefore investigated.

\section{Prospective study}

To analyze a potential adverse relationship between length of formalin fixation and DNA histograms and CV, non-tumorous liver tissue were fixed in $10 \%$ neutral buffered formalin for three days, one week, two weeks, three weeks, and four weeks prior to analysis. Liver tissue was obtained from four patients that underwent hepatic resection for HCC in March of 1992 . Immediately after hepatic resection the tissue was cut into small pieces, and five samples of approximately $2 \mathrm{gm}$ wet weight from each patient was fixed in 10\% formalin and embedded in paraffin for the different fixation durations as described above. To analyze the effect of formalin fixation itself, liver tissue which showed a clear single GOG1 peak were analyzed independently of HCC tissue. Histograms of the latter potentially exhibit multiple DNA ploidy patterns. Fresh frozen samples $\left(-80^{\circ} \mathrm{C}\right)$ without formalin fixation were used as controls. In the control samples, nuclei were obtained by detergent treatment according to the method of Sasaki et al [12].

\section{Statistical analysis}

Statistical analyses were performed using Students' $t$ test and correlation coefficient analysis. A p value of $<0.05$ was considered statistically significant.
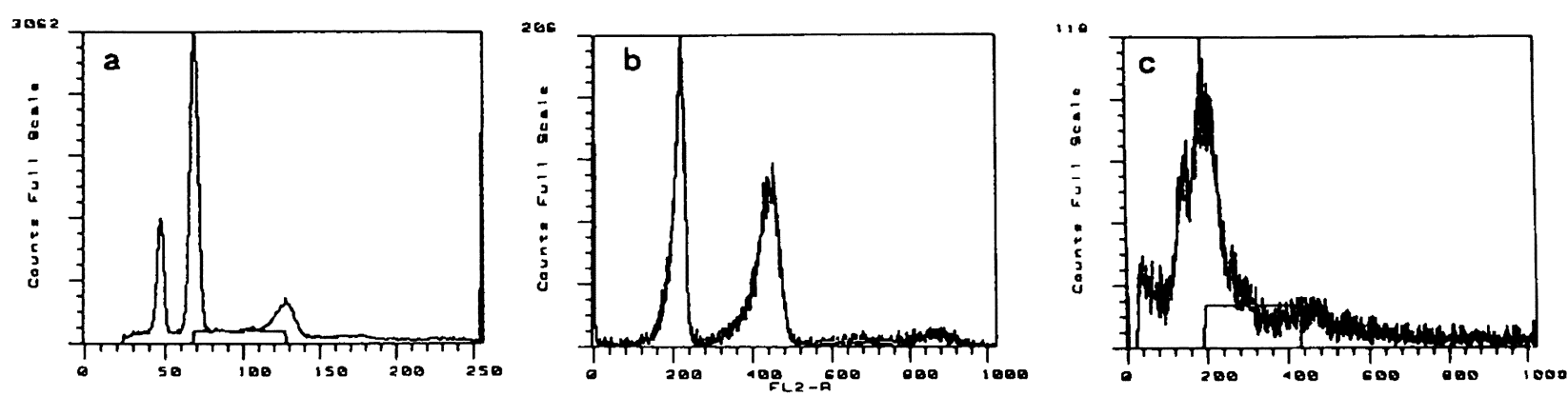

Fig. 1. Examples of flow cytometry DNA histograms showing DNA ploidy patterns. a: $C V=4.6, \mathrm{DNA}$ Index $(\mathrm{DI})=1.4, \quad \mathrm{~b}: \mathrm{CV}=6.8$, $\mathrm{DI}=1.8$, c: $\mathrm{CV}=13.3$, DI cannot be calculated. $\mathrm{CV}$, coefficient of variation.

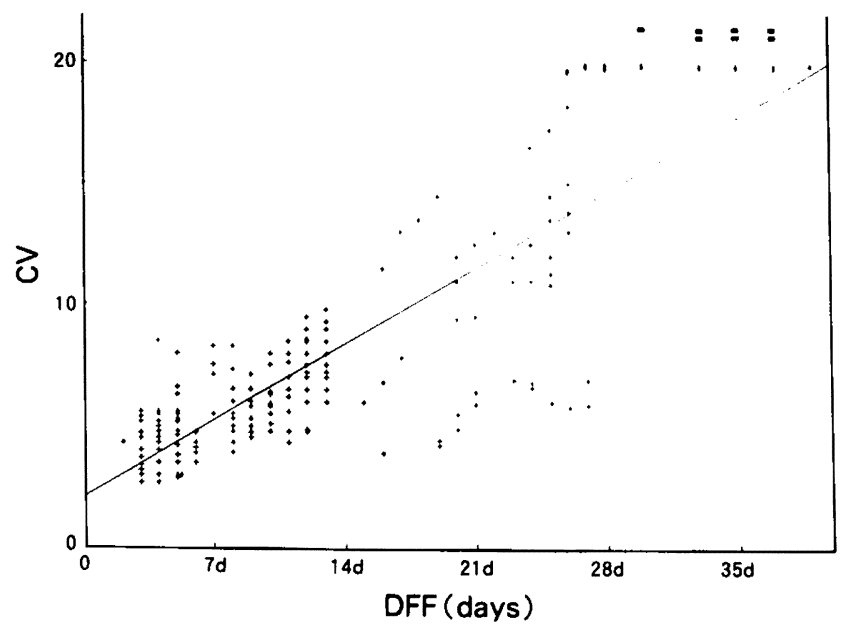

Fig. 2. Relationship between CV and DFF. CV plotted as DFF of samples of which $\mathrm{CV}$ is below 20.0: $(+)$, and uninterpretable cases: $(-),(r=0.69, p<0.001$ defined by the equation, $y=0.454 x+2.02)$. Uninterpretable cases were excluded from the linear correlative analysis. DFF, duration of formalin fixation.

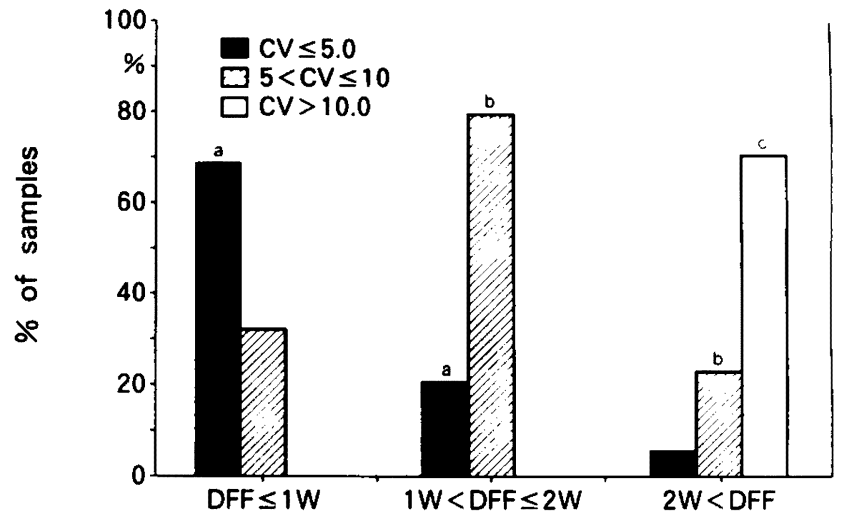

Fig. 3. Summary of the correlation between CV and DFF. Samples in which the $\mathrm{CV}$ was less than 5.0 were significantly more frequent when the DFF $<1$ week (a: $p<0.01$ ); samples in which the $C V$ was between 5 and 10 , were most frequently seen when the DFF was greater than one week (b: $p<0.01$ ); samples in which the CV was greater than 10.0 were noted when the DFF exceeded two weeks (c: $\mathrm{p}<0.001)$. 

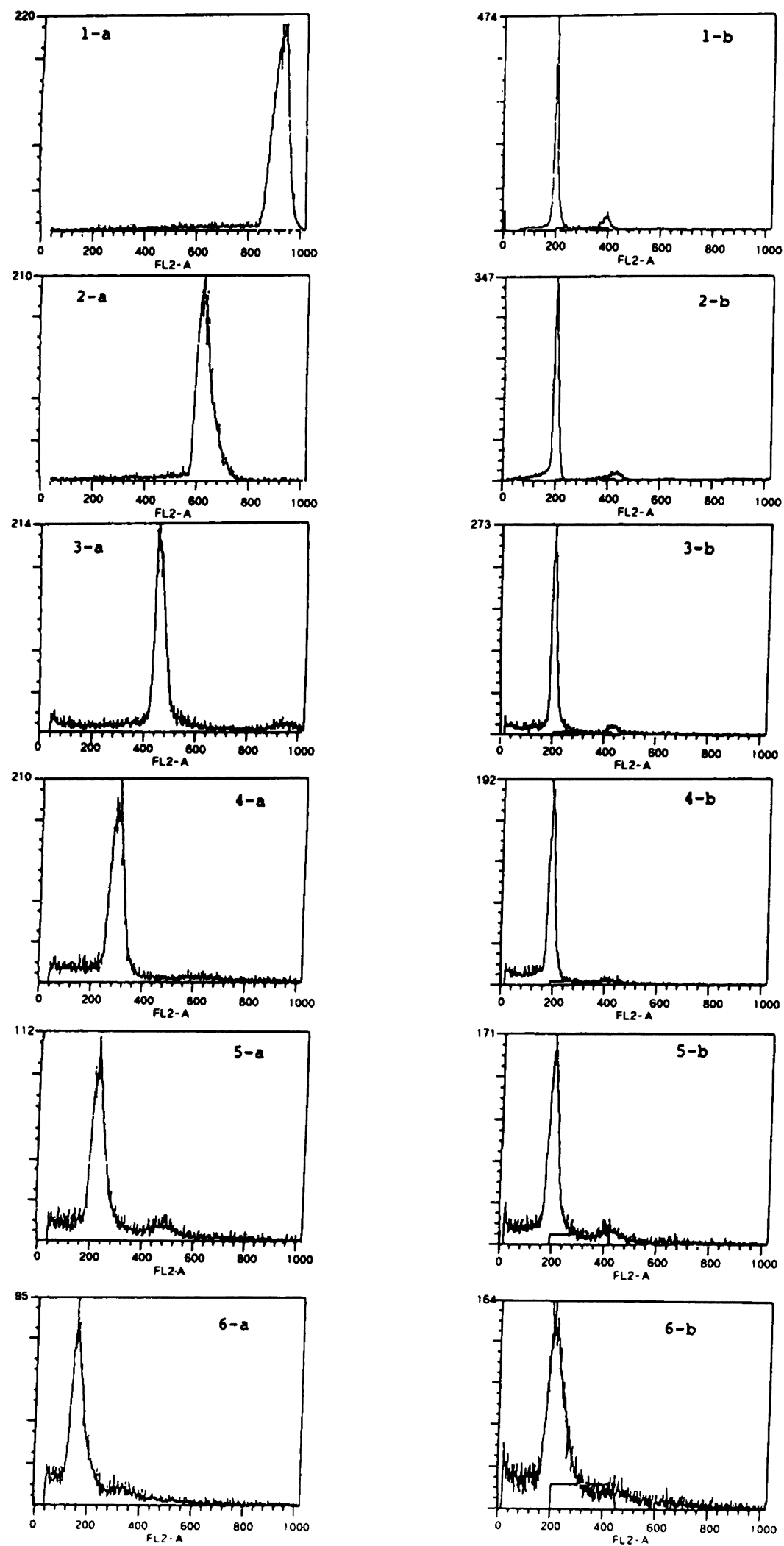

Fig. 4. Prospective analysis of flow cytometry DNA histograms as a function of fixation procedure. 1, unfixed (fresh frozen); 2 , three days' DFF; 3, one week's DFF; 4, two weeks' DFF; 5, three weeks' DFF; 6, four weeks' DFF. Series 1-a to 6-a were obtained by fixing fluorescence (ie, unfixed sample was used as control); series 1-b to 6-b were obtained by fixing G0G1 peak channel number. 


\section{Results}

\section{Retrospective study}

Of the 174 cases, 128 showed a CV of less than 10.0, and therefore could be analyzed using a DNA histogram. Forty-six samples showed a CV of more than 10.0, and were, therefore, inadequate for data analysis. Seven of the 46 were classified as uninterpretable. Representative examples of DNA ploidy patterns are shown in Figure 1. Among 128 interpretable cases, 68 (53\%) were judged to be aneuploid and $60(47 \%)$ diploid. Figure 2 shows the $\mathrm{CV}$ as a function of the DFF. All of the samples in which the DFF was less than two weeks showed a CV under 10.0. In contrast, a broad G0G1 peak in which the $\mathrm{CV}$ was more than 10.0, occurred frequently when the DFF was greater than two weeks. There was a significant linear correlation between the CV and DFF $(r=0.69, p<0.001)$. A summary of the data in which samples were divided into three groups by DFF (i.e., less than one week, from one to two weeks, and greater than two weeks), is presented in Figure 3. Each group was divided into a CV of less than 5 , from 5-10, or greater than 10 . The percentage of CV less than 5.0 was significantly greater $(p<0.01)$ in the samples in which the DFF was less than one week. The percentage with a CV between 5 and 10 was significantly greater $(p<0.01)$ in those samples fixed for one to two weeks, and the proportion of samples with a CV greater than 10.0 was significantly greater $(p<0.001)$ in samples fixed for more than two weeks.

\section{Prospective study}

As shown in Figure 4, a decrease in the fluorescence intensity was observed as the DFF increased. There was a progressive increase in the baseline artifact and the G0G1 peak widened with prolonged fixation. In comparison to fresh frozen materials, the mean relative fluorescence intensity in four formalin fixed tissues decreased as the DFF became prolonged, (three days; 70.0 $\pm 1.0 \%, \mathrm{M} \pm$

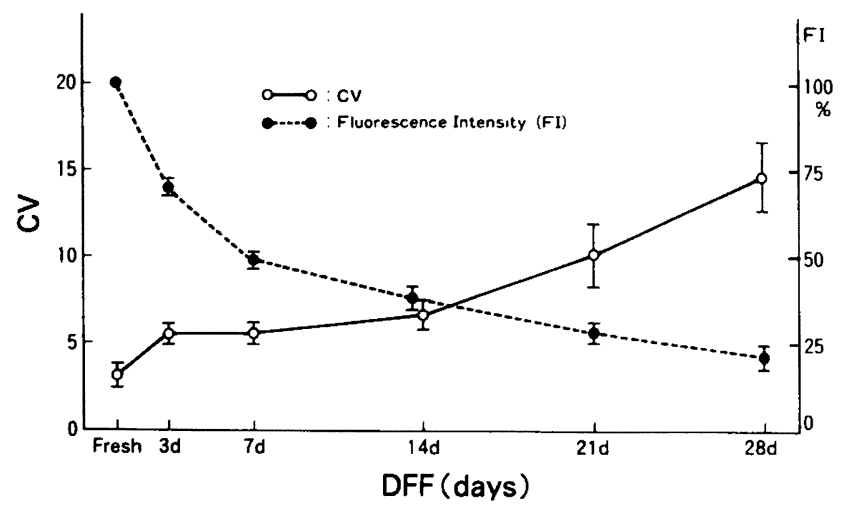

Fig. 5. Changes in fluorescence intensity and $\mathrm{CV}$ as a function of duration of formalin fixation. The fluorescence intensity decreased and the $C V$ increased as the DFF became prolonged, $M \pm S$.D. The relative fluorescence intensity is expressed as the ratio of peak fluorescence in fresh versus formalin fixed samples.
S.D.; 1 week, $49.5 \pm 1.3 \%, 2$ weeks, $38.1 \pm 4.3 \%, 3$ weeks, $29.2 \pm 4.1 \%$, and 4 weeks, $21.2 \pm 6.2 \%$ ). The mean $C V$ increased following formalin fixation (i.e., fresh, $3.1 \pm 0.27 ; 3$ days, $5.5 \pm 0.56 ; 1$ week, $5.4 \pm 0.41 ; 2$ weeks, $6.8 \pm 0.98 ; 3$ weeks, $10.1 \pm 1.95 ; 4$ weeks, $14.7 \pm 3.57$ ), and was greater than 10.0 when the DFF exceeded two weeks (Fig. 5).

\section{Discussion}

Formalin fixation cross-links proteins and stabilizes single-stranded DNA [4]. The use of pepsin digestion as described by Hedley et al. [7] serves two purposes. First, it disaggregates and improves the CV of the histograms. In addition, cross-linkage between DNA and nucleoproteins is reduced [7]. Our data, however, suggest that this method is ineffective for samples subjected to prolonged formalin fixation.

Schutte et al. [12] have reported that fluorescence intensity in chicken red blood cells decreased following formalin fixation. The fluorescence intensity of nuclei appered to be less intense and more variable as compared to fresh, unfixed materials. As the mechanisms of how formalin decrease fluorescense by fixing the tissue have not been clarified, it might be possible that formalin has a cross-linkage activity with the double strand DNA, as propidium iodide could not bind to DNA [1]. Esteban $e t$ al. [2] using paraffin-embedded tissues, observed that an increased length of fixation in formalin (i.e., 8, 24, and $48 \mathrm{hr}$ ) resulted in a slight shift to the left in the G0G1 peaks. In this report, we tested the adverse effect of prolonged formalin fixation in paraffin-embedded tissues in a larger series and for an increased length of time.

Our results show that the baseline artifact increased, and the G0G1 peak widened as the DFF increased. This phenomenon was observed in section samples in which nuclei were transected [14]. Production of nuclear debris may increase during sample disaggregation if the nuclei are broken by prolonged formalin fixation.

Despite a DFF of more than two weeks, some samples showed good CV. We cannot completely explain this apparent discrepancy. It is possible, however, that the rate of formalin infiltration may vary between samples. This variation may result from the fact that, 1) some HCC is not encapsulated, 2) the tumor size can vary from $1.0 \mathrm{~cm}$ to more than $20 \mathrm{~cm}$ in diameter and 3) some tumors were fixed without cutting. Tissue in which the HCC is encapsulated, is large in size, or is not cut at the time of fixation will exhibit a slower rate of formalin infiltration. It has not been reported to the detail relationship between the formalin fixation and $\mathrm{CV}$ in various organs. We have analyzed DNA ploidy in various organs including neuroblastoma [10], colon cancer, esophageal cancer and normal esopagus, pancreatic cancer. In these specimens, it was hard to perform DNA ploidy study for the tissue which has been fixed more than 2 weeks because of wide CV.

Fresh samples provide sufficient CV for DNA 
analysis, but their routine use is limited by the inability of research or clinical laboratories to maintain multiple fresh frozen samples. Flow cytometric analysis from paraffinembedded tissue still has excellent merits. First, embedded tissue is easily maintained in the laboratory, and retrospective analyses are possible. In addition, the sample can be chosen after pathological investigation, and multiple samples can be analyzed at one time. Finally, the sample can be easily stored at room temperature and transported from laboratory to laboratory.

In summary, we believe that flow cytometric analysis from paraffin-embedded materials is a powerful technique which provides useful information if the length of formalin fixation is not prolonged. The duration of formalin fixation should be limited to two weeks prior to embedding the sample in paraffin.

\section{References}

1. Darzynkiewics, Z.: Conformation of RNA in situ as studied by acridine orange staining and automated cytofluorometry. Exp. Cell Res. 95: 143-147, 1975.

2. Esteban, J. M., Shaibani, K., Owens, M., Joyce, J., Bailey, A. and Battifora, H.: Effects of various fixatives and fixation conditions on DNA ploidy analysis. A need for strict internal DNA standards. Am. J. Clin. Pathol. 95: 460-466, 1991.

3. Fossa, S. D., Berner, A., Wahre, H., Heien, T., Juul, M. E. H., Ouden, D., Pettersen, E. O., Wang, N. and Tribukait, B.: DNA ploidy in cell nuclei from paraffin-embedded materialcomparison of results from two laboratories. Cytometry 13: 395-403, 1992.

4. Fraschini, A., Pellicciari, C., Biggiogera, M. and Manfredi Romanini, M. G.: The effect of different fixatives on chromatin: Cytochemical and ultrastructural approaches. Histochem. J. 13: 763-779, 1981.

5. Fujimoto, J., Okamoto, E., Yamanaka, N., Mitunobu, M. and
Toyosaka, A.: Flow cytometric DNA analysis of hepatocellular carcinoma. Cancer 67: 939-944, 1991.

6. Giorgi, J. V., Burtubise, P. E., Cram, L. S., Parker, J. W. and La Via, M.F.: Clinical applications of cytometry: 6th annual meeting. Cytometry 13: 445-447, 1992.

7. Hedley, D. W., Friedlander, M. L., Taylor, I. W., Rugg, C. A. and Musgrove, E. A.: Method for analysis of cellular DNA content of paraffin-embedded pathological material using flow cytometry. J. Histochem. Cytochem. 31: 1333-1335, 1983.

8. Kute, T. E., Galleshaw, G. J., Hopkins, M., Buss, D. and Case, D.: How reproducible are flow cytometry data from paraffinembedded blocks? Cytometry 9: 494-498, 1988.

9. Merkel, D. E. and McGuire, W. L.: Ploidy, proliferative activity and prognosis. Cancer 65: 1194-1205, 1990.

10. Muraji, T., Okamoto, E., Fujimoto, J., Suita, S. and Nakagawara, A.: Combined determination of $\mathrm{N}$-myc oncogene amplification and DNA ploidy in neuroblastoma. Cancer 72: 2863-2768, 1993.

11. Sakaki, K., Hashimoto, T., Kawachino, K. and Takahashi, M.: Intratumoral regional differences in DNA ploidy of gastrointestinal carcinomas. Cancer 62: 2569-2575, 1988.

12. Schutte, B., Reynders, M. J., Bosman, F. T. and Blijham, G. H.: Flow cytometric determination of DNA ploidy level in nuclei isolated from paraffin-embedded tissue. Cytometry 5: 26-29, 1985.

13. Sigudsson, H., Baldetorp, B., Borg, A., Dalberg, M., Ferno, M., Killander, D. and Olsson, H.: Indicators of prognosis in node-negative breast cancer. N. Engl. J. Med. 322: 1045-1053, 1990.

14. Stephenson, R. A., Gay, H., Fair, W. R. and Melamed, M. R.: Effect of section thickness on quality of flow cytometric DNA content determinations in paraffin-embedded tissue. Cytometry 7: 41-44, 1986.

15. Taylor, S. R. and Locker, J.: A comparative analysis of nuclear DNA content and $\mathrm{N}$-myc gene amplification in neuroblastoma. Cancer 65: 1360-1366, 1990.

16. Vindelov, L. L., Christensen, I. J. and Nissen, N. I.: A detergent-trypsin method for preparation of nuclei for flow cytometric DNA analysis. Cytometry 3: 232-237, 1983. 